\title{
Structural Identification of Caged Vanadium Doped Silicon Clusters
}

\author{
P. Claes, ${ }^{1}$ E. Janssens, ${ }^{1, *}$ V. T. Ngan, ${ }^{2}$ P. Gruene, ${ }^{3}$ J. T. Lyon, ${ }^{3}$ D. J. Harding, ${ }^{3}$ A. Fielicke, ${ }^{3}$ \\ M. T. Nguyen, ${ }^{2}$ and P. Lievens ${ }^{1}$ \\ ${ }^{1}$ Laboratory of Solid State Physics and Magnetism, Katholieke Universiteit Leuven, \\ Celestijnenlaan 200D, B-3001 Leuven, Belgium \\ ${ }^{2}$ Department of Chemistry, Katholieke Universiteit Leuven, Celestijnenlaan 200F, B-3001 Leuven, Belgium \\ ${ }^{3}$ Fritz-Haber-Institut der Max-Planck-Gesellschaft, Faradayweg 4-6, D-14195 Berlin, Germany
}

(Received 6 April 2011; published 17 October 2011)

\begin{abstract}
The geometry of cationic silicon clusters doped with vanadium, $\mathrm{Si}_{n} \mathrm{~V}^{+}(n=12-16)$, is investigated by using infrared multiple photon dissociation of the corresponding rare gas complexes in combination with $a b$ initio calculations. It is shown that the clusters are endohedral cages, and evidence is provided that $\mathrm{Si}_{16} \mathrm{~V}^{+}$is a fluxional system with a symmetric Frank-Kasper geometry.
\end{abstract}

The ongoing trend towards further miniaturization in microelectronics triggers a quest for nanostructured building blocks. Given the importance of silicon in the semiconductor industry, it is straightforward to consider small silicon particles for nanostructuring. The search for silicon building blocks was initiated in the 1990s and revealed strong size dependencies and cluster geometries that do not correspond to bulk fragments [1]. Unfortunately, elemental silicon clusters have dangling bonds, which renders them chemically reactive and therefore not suitable as nanoscale building blocks [2]. In contrast to carbon, silicon prefers the formation of $\sigma$ bonds through $s p^{3}$ hybridization, resulting in three-dimensional structures [3]. Ion mobility studies demonstrated that silicon structures follow a prolate growth sequence and no fullerenelike caged particles are formed $[4,5]$. However, incorporating a metal atom or hydrogenation can saturate the dangling bonds and induce the formation of caged silicon clusters $[3,6,7]$.

Theoretical investigations of doped silicon clusters have considered dopants from almost every group of the periodic table [6]. Most interestingly, it is predicted that transition-metal atoms possibly stabilize the clusters and induce the formation of symmetric endohedral cages with the dopant atom at the center of the cage, which is of relevance for novel silicon based nanostructured devices [8-12]. For example, fullerenelike cages and Frank-Kasper (FK) polyhedrons, which are tetrahedrally close-packed structures containing interpenetrating polyhedra with coordination numbers $12,14,15$, or 16 [13], are predicted for $\mathrm{Ti}$ and $\mathrm{V}$ doped silicon clusters with at least $12 \mathrm{Si}$ atoms $[10,14,15]$. However, if there are insufficient $\mathrm{Si}$ atoms to fully enclose the dopant atom, basketlike structures are formed [11]. Although mass spectrometry and photodissociation experiments $[8,16-19]$ show an enhanced stability of specific transition-metal doped silicon clusters, no single experiment has yet provided detailed information on their structure. Up to now, mainly indirect evidence is found for the formation of symmetric species by photoelectron and x-ray spectroscopy studies [20-22] and chemical probe methods [20,23].

In this Letter, the structure of size selected endohedrally doped silicon clusters is obtained by combining experimental infrared multiple photon dissociation (IR-MPD) spectroscopy with quantum chemical calculations. It will be shown that the $\mathrm{V}$ dopant atom in the cationic $\mathrm{Si}_{n} \mathrm{~V}^{+}$ $(n=12-16)$ clusters locates at the center of a cage. Moreover, it is demonstrated that $\mathrm{Si}_{16} \mathrm{~V}^{+}$is a fluxional cluster, oscillating around a symmetric FK polyhedral transition state.

The experiments are performed in a molecular beam setup, which contains a dual laser vaporization source [24] and a time-of-flight mass spectrometer [25]. The setup is connected to a beam line of the Free Electron Laser for Infrared eXperiments (FELIX) [26]. Source parameters are optimized for the formation of cold $(100 \mathrm{~K})$ singly vanadium doped silicon clusters. The formation of clusterxenon complexes is induced by addition of a fraction $(0.5 \%)$ of isotopically enriched ${ }^{129} \mathrm{Xe}$ to the $\mathrm{He}$ carrier gas. Resonant absorption of IR photons and subsequent vibrational energy redistribution heat the clusters and may result in evaporation of the weakly bound Xe atom. The IRMPD depletion spectra are constructed by recording the ion intensities of the cluster-xenon complexes as a function of the FELIX energy $\left(160-550 \mathrm{~cm}^{-1}\right.$ range, linewidth of $\left.3-4 \mathrm{~cm}^{-1}\right)$. From the depletion spectra, IR absorption spectra are calculated as described previously [25].

Quantum chemical computations are carried out with GAUSSIAN 03 [27]. Geometry optimizations are performed by using the pure BP86 functional $[28,29]$, in conjunction with the 6-311+G(d) basis set. This methodology was successful in evaluating IR spectra of bare $\mathrm{Si}_{n}^{+}(n=$ 6-21) [30], $\mathrm{Si}_{n}^{0}(n=6,7,10)$ [31], and exohedrally doped $\mathrm{Si}_{n} X^{+}(X=\mathrm{V}, \mathrm{Cu} ; n=4-11)$ clusters [32,33]. Initial atomic configurations are selected on the basis of structures reported in literature and by substitution or addition of a V atom to bare silicon clusters [30]. The plotted IR 
spectra are based on calculated harmonic vibrational frequencies using Gaussian line width broadening $\left(5 \mathrm{~cm}^{-1}\right.$ FWHM) and by scaling the frequencies with a constant multiplication factor of 1.03 , consistent with our earlier work [30-33]. Structural assignment is done by comparing the IR-MPD spectra with calculated vibrational spectra of the lowest lying isomers.

The experimental IR-MPD spectra of $\mathrm{Si}_{n} \mathrm{~V}^{+}$ $(n=12-15)$ are presented in Fig. 1 together with the computed harmonic vibrational spectra of the lowest energy isomers found in the calculations. An earlier experiment demonstrated that $\mathrm{Ar}$ physisorption provides structural information because $\mathrm{Ar}$ attaches only to surface-located transition-metal atoms and not to elemental silicon clusters. $\mathrm{Si}_{11} \mathrm{~V}^{+}$was shown to be the critical size for Ar physisorption and endohedral structures are expected from $\mathrm{Si}_{12} \mathrm{~V}^{+}$onwards [23]. A detailed comparison of the IR-MPD spectra with computed spectra for different $\mathrm{Si}_{n} \mathrm{~V}^{+} \quad(n=12-16)$ isomers can be found in the Supplemental Materials [34]. For all investigated sizes the experimental IR-MPD spectra agree best with the harmonic vibrational spectra of the lowest energy isomer found at the BP86/6-311+G(d) level.

For $\mathrm{Si}_{12} \mathrm{~V}^{+}$, both bands at the low frequency end of the spectrum (200 and $225 \mathrm{~cm}^{-1}$ ) and the two groups of

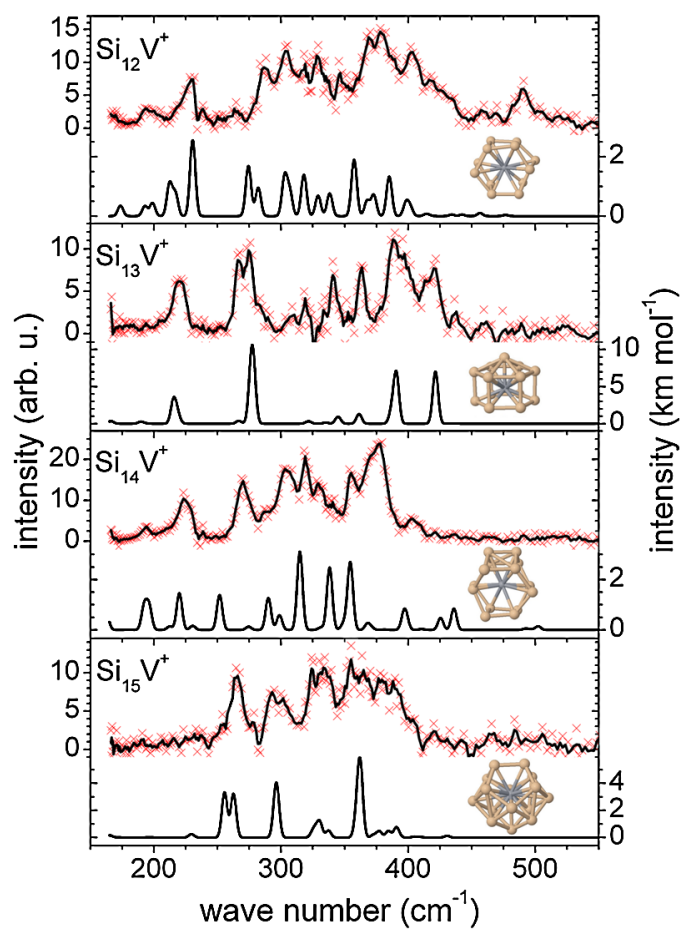

FIG. 1 (color online). IR-MPD spectra (upper traces) of $\mathrm{Si}_{n} \mathrm{~V}^{+} \cdot$ Xe complexes $(n=12-15)$ and the corresponding computed harmonic vibrational spectra and geometries of the obtained lowest energy isomers of $\mathrm{Si}_{n} \mathrm{~V}^{+}$(lower traces). The experimental data points (red crosses) are overlaid with a three-point running average. absorption bands around 310 and $390 \mathrm{~cm}^{-1}$ are reproduced in the calculated vibrational spectrum of a distorted hexagonal prism (DHP) in a singlet electronic state. However, the calculated intensity of the band at $475 \mathrm{~cm}^{-1}$ is less intense than the IR-MPD band at $490 \mathrm{~cm}^{-1}$. The singlet DHP, which also is the putative ground state of the isoelectronic $\mathrm{Si}_{12} \mathrm{Ti}[11,35,36]$, is the lowest energy isomer found at the BP86/6-311+G(d) level. Other isomers, such as a triplet DHP and basket-shaped structures, are at least $0.26 \mathrm{eV}$ less stable [34]. For the intense absorption bands at $360-380 \mathrm{~cm}^{-1}, 75 \%$ of the complex intensity is depleted by FELIX. This sets the upper limit for the relative abundance of other isomers. Therefore, the singlet DHP is assigned as the most abundant $\mathrm{Si}_{12} \mathrm{~V}^{+}$isomer in the cluster beam.

For $\mathrm{Si}_{13} \mathrm{~V}^{+}$, also a spin singlet was located as the ground state. The cluster has $C_{6 v}$ symmetry, consisting of a hexagonal prism whose hexagonal face is capped by a $\mathrm{Si}$ atom. The same structure was predicted for the isovalent $\mathrm{Si}_{13} \mathrm{Ti}$ $[11,37]$. The calculated vibrational spectrum of the lowest energy isomer of $\mathrm{Si}_{13} \mathrm{~V}^{+}$matches the IR-MPD spectrum of the corresponding Xe complex well. Calculated doubly degenerate modes at 216,276 , and $389 \mathrm{~cm}^{-1}$ seem to be split in the experiment. This splitting is likely caused by the Xe adsorption, which distorts the $C_{6 v}$ symmetry. However, the experimentally observed splitting $\left(5-8 \mathrm{~cm}^{-1}\right)$ is larger than the calculated one $\left(1-2 \mathrm{~cm}^{-1}\right)$. High energy modes at 434, 438, and $461 \mathrm{~cm}^{-1}$ are IR inactive when neglecting the messenger atom but become active when a Xe ligand is attached.

The IR-MPD spectrum of $\mathrm{Si}_{14} \mathrm{~V}^{+} \cdot$ Xe shows characteristic absorptions at 224 and $270 \mathrm{~cm}^{-1}$ and groups of bands in the $295-335$ and $350-410 \mathrm{~cm}^{-1}$ ranges. Computationally, several isomers of $\mathrm{Si}_{14} \mathrm{~V}^{+}$were located close in energy (five isomers within $0.1 \mathrm{eV}$ ) [34]. In line with an earlier study [15], most low energy isomers have singlet electronic states and are constructed by addition of two $\mathrm{Si}$ atoms to various sites of a DHP and subsequent structural relaxation. Although the lowest energy isomer (shown in Fig. 1) explains certain features of the IR-MPD spectra and none of the other isomers found matches the experiment better [34], the agreement is not conclusive. It is not certain that the currently found DHP based ground state is the main $\mathrm{Si}_{14} \mathrm{~V}^{+}$isomer produced in the experiment.

The absorption bands in the IR-MPD spectrum of $\mathrm{Si}_{15} \mathrm{~V}^{+} \cdot \mathrm{Xe}$ match the vibrational modes of a structure based on a combination of 2 pentagons and 10 rhombuses, which was located as the ground state in agreement with earlier predictions for $\mathrm{Si}_{15} \mathrm{~V}^{+}[10,15]$ and the isoelectronic $\mathrm{Si}_{15} \mathrm{Ti}[15,37]$. Vibrational spectra of hexagonal prism based structures, such as found in some studies [38], do not match the IR-MPD spectrum [34].

The IR-MPD spectrum of $\mathrm{Si}_{16} \mathrm{~V}^{+} \cdot \mathrm{Xe}$ is very different from those of $\mathrm{Si}_{n} \mathrm{~V}^{+} \cdot \mathrm{Xe}(n=12-15)$. It shows no distinct, narrow absorption peaks but has a couple of broad 
features around $360 \mathrm{~cm}^{-1}$ and below $300 \mathrm{~cm}^{-1}$ (see Fig. 2). The lower signal-to-noise ratio of this spectrum is caused by a small $\mathrm{Si}_{16} \mathrm{~V}^{+} \cdot \mathrm{Xe}$ mass spectrometric signal, indicating a low Xe absorption probability.

$\mathrm{Si}_{16} \mathrm{~V}^{+}$is one of the best studied endohedrally doped silicon clusters in the literature, mainly because of its enhanced stability indicated by its large abundance in the $\mathrm{Si}_{n} \mathrm{~V}^{+}$mass spectrum [17]. $\mathrm{Si}_{16} \mathrm{~V}^{+}$is predicted to have a symmetric FK polyhedral structure [10,15,39]. Its stability was rationalized by means of an electron counting rule. Each $\mathrm{Si}$ atom is bonded with the $\mathrm{V}$ dopant and donates one electron to the valence manifold. With the five valence electrons of $\mathrm{V}$ and the cationic state of the cluster, this results in a 20 electron closed shell system [10].

Our calculations find the highly symmetric FK polyhedron to be a transition state (TS- $\left.T_{d}\right)$. Following the displacement vectors of the imaginary frequencies in either direction leads to a slightly distorted caged structure iso1- $T$, lying $0.05 \mathrm{eV}$ lower in energy. The calculated spectrum of iso1- $T$ has one intense mode at $370 \mathrm{~cm}^{-1}$, which agrees well with the position of the experimental band center but does not explain the width of the band. The geometries of both TS- $T_{d}$ and iso1- $T$ consist of an inner tetrahedral shell with four $\mathrm{Si}$ atoms $(\mathrm{Si}-\mathrm{V}$ distances of 2.541 and $2.545 \AA$ for $T_{d}$ and $T$, respectively) and an outer shell with $12 \mathrm{Si}$ atoms (Si-V distances of 2.829 and $2.836 \AA$ for $T_{d}$ and $T$, respectively). Relaxing from $T_{d}$ to $T$

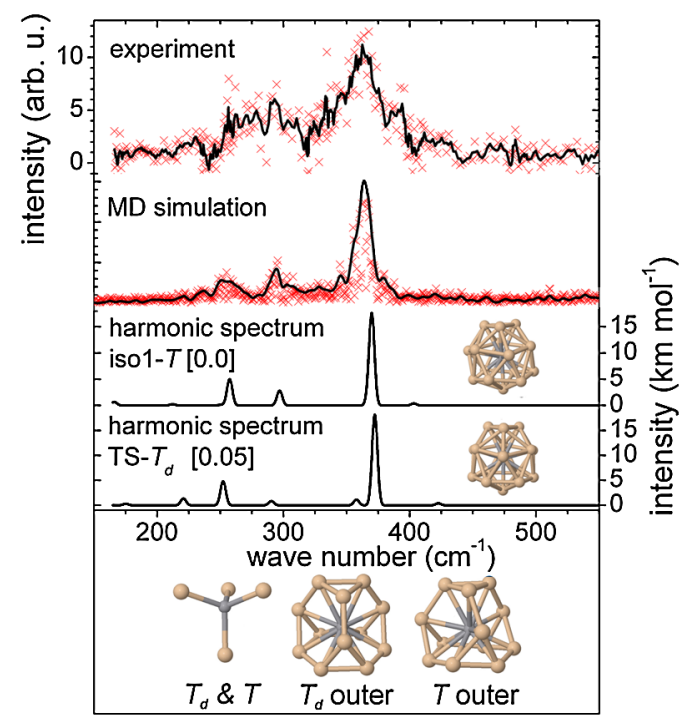

FIG. 2 (color online). IR-MPD spectrum of $\mathrm{Si}_{16} \mathrm{~V}^{+} \cdot \mathrm{Xe}$ (upper trace). The 2 nd trace shows the IR spectrum obtained from the MD simulation starting from iso1- $T$ (red crosses) and a spectrum that is obtained by overlaying the discrete points with a Gaussian profile $\left(5 \mathrm{~cm}^{-1}\right.$ FWHM) to account for the FELIX linewidth (black line). Computed harmonic vibrational spectra of iso1- $T$ and $\mathbf{T S}-T_{d}$ are given in the $3 \mathrm{rd}$ and 4 th traces. Relative energies in $\mathrm{eV}$ at the BP86/6-311+G(d) level are given in brackets. The structures shown in the lower trace represent the inner tetrahedral and outer shells of TS- $T_{d}$ and iso1- $T$. symmetry, the inner shell remains essentially unchanged while the outer shell is distorted at the symmetrical planes (see structures in the lower trace of Fig. 2).

The small barrier over TS- $T_{d}$ between degenerate iso1- $T$ states suggests that fluxional rearrangements, i.e., rapid transitions between chemically equivalent structures [3], may be possible. A similar fluxional behavior for $\mathrm{Si}_{6}$ $[31,40]$ lead to a broadening of the IR absorption bands. We have investigated the possibility that dynamic and anharmonic effects are responsible for the band broadening in the IR-MPD spectrum of $\mathrm{Si}_{16} \mathrm{~V}^{+}$using a molecular dynamics (MD) simulation performed with the FHI-AIMS program [41,42]. An IR spectrum is obtained from the MD trajectory as the Fourier transform of the autocorrelation function of the time-dependent dipole moment [43]. Details of the calculations are given in the Supplemental Materials [34]. The spectrum of $\mathrm{Si}_{16} \mathrm{~V}^{+}$calculated this way (see 2nd trance of Fig. 2) shows a significant degree of broadening. For comparison, we have calculated a spectrum for $\mathrm{Si}_{8} \mathrm{~V}^{+}$using the same method, which did not show such broadening. Analysis of the MD trajectory supports the interpretation of $\mathrm{Si}_{16} \mathrm{~V}^{+}$being dynamic, undergoing rapid transitions between nearly degenerate local minima. A more detailed analysis of its dynamics will be presented elsewhere.

We conclude that, using the IR-MPD technique on cluster-xenon complexes in combination with $a b$ initio calculations, the geometry of $\mathrm{Si}_{n} \mathrm{~V}^{+}(n=12-16)$ in the gas phase could be identified. The clusters have endohedral caged structures, stabilized by the V dopant atom. For all sizes the experimental IR-MPD spectra agree best with the lowest energy isomer found at the BP86/6-311+G(d) level. However, higher energy isomers may, to some extent, be present in the molecular beam and therefore contribute to the experimental IR spectra. $\mathrm{Si}_{12} \mathrm{~V}^{+}, \mathrm{Si}_{13} \mathrm{~V}^{+}$, and presumably $\mathrm{Si}_{14} \mathrm{~V}^{+}$have hexagonal prism based structures. For the latter, however, the assignment is not conclusive. $\mathrm{Si}_{15} \mathrm{~V}^{+}$is found to be built up from pentagons and rhombuses. $\mathrm{Si}_{16} \mathrm{~V}^{+}$, finally, was shown to have a fluxional behavior resulting from rapid interchange of the atomic positions between a slightly distorted and a perfect FrankKasper polyhedral geometry corresponding to the ground state and a transition state, respectively.

The authors gratefully acknowledge the support of the Stichting voor Fundamenteel Onderzoek der Materie (FOM) in providing beam time on FELIX and highly appreciate the skillful assistance of the FELIX staff. D. J.H. and A.F. thank Luca Ghiringhelli and Matthias Scheffler for helpful discussions. This work is supported by the European Community's FP7/2007-2013 (Grant No. 226716), the Research Foundation-Flanders (FWO), the Flemish Concerted Action (GOA), the Belgian Interuniversity Poles of Attraction (IAP), and the Deutsche Forschungsgemeinschaft within FOR 1282 (FI $893 / 4-1)$. P. C. is grateful for the financial support by the 
IWT-Vlaanderen. J. T. L. and D. J. H. are grateful for funding from the Alexander von Humboldt Foundation.

*ewald.janssens@fys.kuleuven.be

[1] K. M. Ho, A. A. Shvartsburg, B. Pan, Z. Y. Lu, C. Z. Wang, J. G. Wacker, J. L. Fye, and M. F. Jarrold, Nature (London) 392, 582 (1998)

[2] U. Röthlisberger, W. Andreoni, and M. Parrinello, Phys. Rev. Lett. 72, 665 (1994).

[3] A. D. Zdetsis, Phys. Rev. B 76, 075402 (2007).

[4] M. F. Jarrold and V. A. Constant, Phys. Rev. Lett. 67, 2994 (1991).

[5] R. R. Hudgins, M. Imai, M. F. Jarrold, and P. Dugourd, J. Chem. Phys. 111, 7865 (1999).

[6] V. Kumar, in Nanosilicon, edited by V. Kumar (Elsevier, New York, 2007).

[7] N. Veldeman, P. Gruene, A. Fielicke, P. Claes, V. T. Ngan, M. T. Nguyen, and P. Lievens, in The Handbook of Nanophysics: Clusters and Fullerenes, edited by K. D. Sattler (CRC, Boca Raton, FL, 2010).

[8] H. Hiura, T. Miyazaki, and T. Kanayama, Phys. Rev. Lett. 86, 1733 (2001).

[9] S. N. Khanna, B. K. Rao, and P. Jena, Phys. Rev. Lett. 89, 016803 (2002).

[10] J. U. Reveles and S. N. Khanna, Phys. Rev. B 74, 035435 (2006).

[11] H. Kawamura, V. Kumar, and Y. Kawazoe, Phys. Rev. B 71, 075423 (2005)

[12] F. Hagelberg, C. Xiao, and W. A. Lester, Jr., Phys. Rev. B 67, 035426 (2003)

[13] F. C. Frank and J.S. Kasper, Acta Crystallogr. 12, 483 (1959).

[14] V. Kumar, T. M. Briere, and Y. Kawazoe, Phys. Rev. B 68, 155412 (2003).

[15] M. B. Torres, E. M. Fernández, and L. C. Balbás, Phys. Rev. B 75, 205425 (2007).

[16] S. M. Beck, J. Chem. Phys. 87, 4233 (1987).

[17] K. Koyasu, M. Akutsu, M. Mitsui, and A. Nakajima, J. Am. Chem. Soc. 127, 4998 (2005).

[18] S. Neukermans, X. Wang, N. Veldeman, E. Janssens, R. E. Silverans, and P. Lievens, Int. J. Mass Spectrom. 252, 145 (2006).

[19] J. B. Jaeger, T. D. Jaeger, and M. A. Duncan, J. Phys. Chem. A 110, 9310 (2006).

[20] K. Koyasu, J. Atobe, M. Akutsu, M. Mitsui, and A. Nakajima, J. Phys. Chem. A 111, 42 (2007).

[21] A. Grubisic, H. Wang, Y. J. Ko, and K. H. Bowen, J. Chem. Phys. 129, 054302 (2008).

[22] J. T. Lau, K. Hirsch, Ph. Klar, A. Langenberg, F. Lofink, R. Richter, J. Rittmann, M. Vogel, V. Zamudio-Bayer, T.
Möller, and B.v. Issendorff, Phys. Rev. A 79, 053201 (2009).

[23] E. Janssens, P. Gruene, G. Meijer, L. Wöste, P. Lievens, and A. Fielicke, Phys. Rev. Lett. 99, 063401 (2007).

[24] W. Bouwen, P. Thoen, F. Vanhoutte, S. Bouckaert, F. Despa, H. Weidele, R. E. Silverans, and P. Lievens, Rev. Sci. Instrum. 71, 54 (2000).

[25] A. Fielicke, G. von Helden, and G. Meijer, Eur. Phys. J. D 34, 83 (2005).

[26] D. Oepts, A. F. G. van der Meer, and P. W. van Amersfoort, Infrared Phys. Technol. 36, 297 (1995).

[27] GAUSSIAN03, Revision C.02, M. J. Frisch et al., Gaussian, Inc., Wallingford, CT, 2004.

[28] A. D. Becke, Phys. Rev. A 38, 3098 (1988).

[29] J. P. Perdew, Phys. Rev. B 33, 8822 (1986).

[30] J. T. Lyon, P. Gruene, A. Fielicke, G. Meijer, E. Janssens, P. Claes, and P. Lievens, J. Am. Chem. Soc. 131, 1115 (2009).

[31] A. Fielicke, J. T. Lyon, M. Haertelt, G. Meijer, P. Claes, J. De Haeck, and P. Lievens, J. Chem. Phys. 131, 171105 (2009).

[32] P. Gruene, A. Fielicke, G. Meijer, E. Janssens, V. T. Ngan, M. T. Nguyen, and P. Lievens, Chem. Phys. Chem. 9, 703 (2008).

[33] V. T. Ngan, P. Gruene, P. Claes, E. Janssens, A. Fielicke, M. T. Nguyen, and P. Lievens, J. Am. Chem. Soc. 132, 15589 (2010).

[34] See Supplemental Material at http://link.aps.org/ supplemental/10.1103/PhysRevLett.107.173401 for a description of (i) mass spectra of the produced $\mathrm{Si}_{n} \mathrm{~V}^{+}$ clusters, (ii) extended set of structural isomers of $\mathrm{Si}_{n} \mathrm{~V}^{+}$ ( $n=12-16)$ and their vibrational spectra, (iii) influence of the Xe atom on the infrared spectra, and (iv) details of the MD simulation.

[35] J. U. Reveles and S. N. Khanna, Phys. Rev. B 72, 165413 (2005).

[36] J. He, K. Wua, R. Sa, Q. Li, and Y. Wei, Chem. Phys. Lett. 490, 132 (2010).

[37] D. Bandyopadhyay, J. Appl. Phys. 104, 084308 (2008).

[38] J. He, K. Wua, C. Liu, and R. Sa, Chem. Phys. Lett. 483, 30 (2009).

[39] D. Palagin, M. Gramzow, and K. Reuter, J. Chem. Phys. 134, 244705 (2011).

[40] A. D. Zdetsis, J. Chem. Phys. 127, 014314 (2007).

[41] V. Blum, R. Gehrke, F. Hanke, P. Havu, V. Havu, X. Ren, K. Reuter, and M. Scheffler, Comput. Phys. Commun. 180, 2175 (2009).

[42] M. Rossi, V. Blum, P. Kupser, G. von Helden, F. Bierau, K. Pagel, G. Meijer, and M. Scheffler, J. Phys. Chem. Lett. 1, 3465 (2010).

[43] M.P. Gaigeot, M. Martinez, and R. Vuilleumier, Mol. Phys. 105, 2857 (2007). 